\section{Isolation of an Escherichia coli mutant susceptible to a quinolone in an anaerobic environment}

\author{
Eugenio A Debbia, ${ }^{1}$ Anna Marchese ${ }^{1,2}$ \\ ${ }^{1}$ C.A. Romanzi Section of Microbiology, \\ Department of Surgical Sciences and \\ Integrated Diagnostic (DISC), School of \\ Medical and Pharmaceutical Sciences, \\ University of Genoa; ${ }^{2}$ Microbiological \\ Unit, San Martino Polyclinic Hospital, \\ Genoa, Italy
}

\begin{abstract}
Quinolones are bactericidal agents that interfere with the essential prokaryotic enzyme DNA gyrase. While their mechanism of killing appears to be elucidated, one interesting feature is represented by the fact that, under anaerobic conditions, the growth of bacteria is inhibited but their viability is not affected by the first generation of quinolones such as nalidixic acid. More information about the mode of action of these drugs in anaerobiosis might be gained through the availability of strains subjected to enhanced killing in oxygen-deprived media. It has been assumed that when a population of a $\mathrm{AB} 1157\left(\mathrm{~F}^{\prime} / a c\right)$ strain is exposed to nalidixic acid, plasmid-free cells could be recovered from culture treated with sub-inhibitory concentrations of the drug $(2 \mathrm{mg} / \mathrm{L})$ in aerobiosis, and, at the same drug level, only from the rare spontaneous susceptible mutant(s) in anaerobiosis. Extrachromosomal genetic elements, on the contrary, appeared more resistant to these drugs or thermal inactivation of gyrase enzymes either for duplication of genetic materials or for conjugation. ${ }^{5-10}$

Another interesting feature is represented by the fact that, under anaerobic conditions, the growth of bacteria is inhibited but their viability is not affected by the first generation quinolones such as nalidixic acid. ${ }^{3-11}$ However it is reasonable to assume that more information about the mode of action of quinolones in anaerobiosis might be gained through the availability of strains subjected to enhanced killing in oxygendeprived media. Therefore in this paper a method to isolate a strain showing the same susceptibility to nalidixic acid in both aerobic and anaerobic environment is described. A preliminary characterization of the genetic localization of this mutation was also carried out.
\end{abstract} Among plasmid free bacteria found, 1 isolate demonstrated the same MIC value to nalidixic acid in both aerobic and anaerobic conditions. The mutation was co-transferred with $\operatorname{Tn} 10$ inserted at $28.5 \mathrm{~min}$ of the Escherichia coli genetic map into a wildtype strain. These transductants revealed the same phenotypes of the original mutant: susceptibility to nalidixic acid under anaerobic conditions (assessed by time-kill tests) and elongated cells during the aerobic growth, generation time about $65 \mathrm{~min}$ in comparison to $25 \mathrm{~min}$ of the control. Time kill experiment under aerobic environment revealed that the transductant was also susceptible to ciprofloxacin but not nalidixic acid in the presence of chloramphenicol (50 $\mathrm{mg} / \mathrm{L}$ ). These results suggest a possible role of bacterial topoisomerase in the anaerobic susceptibility to nalidixic acid of the mutant.

\section{Introduction}

DNA gyrase and topoisomerase IV are essential enzymes involved in the various phases of bacterial DNA metabolism and gene expression. These proteins are the targets of quinolones, ${ }^{1-3}$ a class of antibacterial agents largely employed in therapy since they have been found to be clinically effective. ${ }^{4}$ Quinolones and temperature-sensitive mutants in both DNA gyrase and topoisomerase IV subunits have been used for a variety of physiological studies. In particular, there is a consistent number of reports showing that chromosome metabolism resulted susceptible to quinolone activity or are affected by mutation that inactive topoisomerase enzymes by temperature.

\section{Materials and Methods}

\section{Bacterial strains}

Strains carrying relevant characteristics for this study are listed in Table 1. Plasmid F'lac (F'128) was one of the F' kit, previously reported ${ }^{10}$. This plasmid carrying the transposon $\mathrm{Tn} 10$ that codes for tetracycline resistance was, it used for strain construction. Transductions were carried out with P1 vir phage. ${ }^{12}$

\section{Media and reagents}

Microorganisms were cultured in LB medium. ${ }^{12}$ Media were supplemented with antibiotics when necessary, nalidixic acid, ciprofloxacin, chloramphenicol and tetracycline were obtained from commercial sources (Sigma, Milan, Italy) and stock
Corrrespondence: Eugenio A. Debbia, C.A Romanzi Section of Microbiology, Department of Surgical Sciences and Integrated Diagnostic (DISC), School of Medical and Pharmaceutical Sciences University of Genoa, Genoa, Italy.

Tel.: +39.010.209.51247.

e-mail: eugenio.debbia@unige.it

Key words: nalidixic acid susceptible, anaerobic environment, bactericidal activity with and without chloramphenicol.

Contributions: the authors contributed equally.

Conflict of interest: the authors declare no potential conflict of interest.

Funding: none.

Received for publication: 25 October 2017. Accepted for publication: 1 December 2017.

This work is licensed under a Creative Commons Attribution NonCommercial 4.0 License (CC BY-NC 4.0).

(C) Copyright E.A Debbia and A. Marchese, 2018 Licensee PAGEPress, Italy

Microbiology Research 2018; 9:7467

doi:10.4081/mr.2018.7467

solutions were prepared following manufacturer's instructions.

\section{Methods for plasmid elimination}

About $5 \times 10^{3} \mathrm{CFU} / \mathrm{ml}$ of log phase of strain studied were cultured in a series of tubes containing $1 \mathrm{ml} \mathrm{LB}$ and graded concentrations of the antibiotic tested. A tube of drug-free LB was used as a control. After incubation for 18-20 h under aerobic and anaerobic conditions at $37^{\circ} \mathrm{C}$ the concentration of drugs that prevent visible bacterial growth were recorded as the MICs. The anaerobic environment was established by culturing the test bacteria for $24 \mathrm{~h}$ in broth covered with $2 \mathrm{~cm}$ of liquid paraffin.

Time-kill experiments were performed by adding the drug at concentrations corresponding to $4 \times \mathrm{MIC}$, to $\log$-phase bacterial cultures diluted to $10^{6}-10^{7} \mathrm{CFU} / \mathrm{ml}$ growing in $500 \mathrm{ml}$ flasks at $37^{\circ} \mathrm{C}$. Just before the compounds were added (zero time) and at 2 , 4 and $6 \mathrm{~h}$ thereafter, bacterial counts were carried out by determining CFU on agar plates. Colonies were counted after $48 \mathrm{~h}$ of incubation at $35^{\circ} \mathrm{C}$. Time-kill under anaerobic conditions were carried out in similar way with the exception that broth culture were covered with $2 \mathrm{~cm}$ of paraffin oil. Bacterial samples were collected using a Hamilton syringe. 


\section{Results}

Considering that the first generation of quinolones, such as nalidixic acid, exhibits an increased MIC values and bacteriostatic activity under anaerobic environment, the detection of these hypothesized hypersensitive mutants in a population where bacterial cells are not dividing cannot be carried out by techniques such as replica plating. To circumvent this problem, it has been assumed the quinolone-susceptible mutant(s) might show the same MIC value $(4 \mathrm{mg} / \mathrm{L})$ for nalidixic acid in both aerobic and anaerobic environment. If this were true, when a population of a AB1157 strain carrying (F'lac::Tn10) is exposed to nalidixic acid, plasmid-free cells could be recovered from culture treated with subinhibitory concentrations of the drug $(2 \mathrm{mg} / \mathrm{L})$ in aerobiosis, and, at the same drug level, only from the rare spontaneous nalidixic acid susceptible mutant(s) in anaerobiosis.

Therefore about $5 \times 10^{3}$ of AB1157 (F'lac::Tn10) bacteria were cultured in a series of tubes containing $1 \mathrm{ml} \mathrm{LB}$ broth and graded concentrations of the nalidixic acid under aerobic and anaerobic conditions. A tube of drug-free broth was used as a control. After incubation for $18-20 \mathrm{~h}$ at $37^{\circ} \mathrm{C}$, bacteria were diluted in saline and plated on drug-free McConkey agar plates scoring for the lactose-negative colonies. Flac free seg- regants were isolated from cultures kept either in anaerobiosis as well as in aerobiosis (Table 1). Putative F'lac::Tn10 cured colonies were picked with sterile wooden toothpicks and gridded on McConkey master plates with and without tetracycline (10 $\mathrm{mg} / \mathrm{L}$ ), for purification, after incubation for 18-20 $\mathrm{h}$ the lactose-negative, tetracycline susceptible colonies, were further collected by toothpick and gridded in quadruplicate in LB agar containing $20 \mathrm{mg} / \mathrm{L}$ of nalidixic acid a plate of drug free medium was used as control. The plates were then incubated under aerobic and anaerobic conditions. The results obtained demonstrated the presence of a nalidixic acid susceptible isolate under anaerobic environment. This strain was collected and tested in broth by macrodilution method for susceptibility to the quinolone in all the experimental conditions. This isolate found exhibited the same MIC (2 mg/L) to nalidixic acid under both aerobic and anaerobic conditions thus it was designed db1771 and considered for further tests.

Phenotypic characterization of this mutant revealed that it grew as filaments mixed with elongated forms which were equivalent in length to 3-5 cells. The generation time was about 65 min in comparison to $25 \mathrm{~min}$ of the control microorganism. In an attempt to identify the chromosomal location of this mutation a complementation analysis was carried out using the F' Kit. It was found that both F' 126 and F' 123 plasmids were able to suppress this mutation restoring the original phenotype to this strain. The F'123 episome includes the trpA-topA-cys $B$ region of the E. coli chromosome- Therefore from the Singer collection we transfer, by $\mathrm{P} 1$ transduction and selecting for tetracycline-resistance, a $\operatorname{Tn} 10$ transposon inserted at $28.5 \mathrm{~min}$ in the $E$. coli map. The donor strain was CAG12028 and the recipient was the strain db1771 that carries the quinolone susceptible mutation under anaerobic environment ( $q s a-1)$. Among the transductants found about $70 \%$ manifested the original phenotype indicating that from the donor strain, together with the transposon, the recipient received the genetic information to correct the qsa-1 mutation. Although these results suggest that the qsa-1 mutation is linked to $28.5 \mathrm{~min}$ of the E. coli genetic map, this particular chromosomal location provides a significative evidence of the possible involvement of the top $A$ gene as responsible of the susceptibility of the strain to nalidixic acid under anaerobic environment. With the help of the Tn10 transposon we tried to transfer in a new cytoplasmic background the qsa-1 gene. To do this P1vir grown on the above mutant db1985 and carrying Tn10 was used to infect a C600 strain selecting for tetracycline resistance. Among the transductants obtained about the $60 \%$ received the $q s a-1$ phenotype of the original mutant. In partic-

Table 1. List of Escherichia coli strains used in this study.

\begin{tabular}{lll} 
Strain & Main characteristics & Source or reference \\
AB1157(F'lac::Tn10) & thr-1, leuB6, proA2, argE3, his-4, thi-1, lac Y1, galK2, rpsL31 Tet-r & Marchese and Debbia, 201610 \\
CAG12028 & MG1655 rci-223::Tn10 & Singer collection ${ }^{13}$ \\
\hline C600 & thr-1, leuB6, thi-1, lac Y1 & Laboratory collection \\
\hline db1771 & AB1157 qsa-1, Tet-s & Spontaneous derivative of AB1157, select lac-, screen Tet-s, this study \\
\hline db1985 & AB1157 Tet-r, qsa-1 & P1(CAG12028) X db1771, select Tet-r, screen qsa-l, this study \\
db1986 & C600 Tet-r, qsa-1 & P1(db1985) X C600, select Tet-r, screen $q s a-1$, this study \\
\hline
\end{tabular}

Tet, tetracycline; s, susceptible; r, resistant; qsa, quinolone susceptible under anaerobic conditions.

Table 2. Incidence of plasmid-loss obtained in E. coli AB1157 (F'lac) treated with nalidixic acid under aerobic and anaerobic conditions.

\begin{tabular}{|c|c|c|c|c|c|c|c|}
\hline & & & Grow & onditions & & & \\
\hline & Aero & & & & Ana & & \\
\hline MIC (mg/L) & Used dose (mg/L) & N.of plasmid loss /total tested & $\%$ & MIC (mg/L) & Used dose $(\mathrm{mg} / \mathrm{L})$ & N.of plasmid loss /total tested & $\%$ \\
\hline 4 & 2 & $130 / 1400$ & 9.3 & 128 & 64 & $14 / 2550$ & 0.5 \\
\hline & 1 & $7 / 585$ & 1.2 & & 32 & $7 / 550$ & 1.3 \\
\hline & & & & 16 & $0 / 122$ & 0 & \\
\hline & & & & 8 & $8 / 1260$ & 0.6 & \\
\hline & & & & 4 & $3 / 800$ & 0.4 & \\
\hline & & & & 2 & $96 / 4500$ & 2.1 & \\
\hline & & & & 1 & $11 / 1800$ & 0.6 & \\
\hline & 0 & & & & 0 & $6 / 900$ & 0.7 \\
\hline
\end{tabular}


ular, in aerobic condition, they exhibited generation time and morphological alteration, as well as nalidixic acid susceptibility under anaerobic environment like the mutant db1771. One randomly chosen of these strains was designated db1986 and used for further studies.

Dynamic bactericidal activity of nalidixic acid and ciprofloxacin with and without chloramphenicol against $q s a-1$ mutant, under aerobic and anaerobic conditions.

The qsa-1 mutant strain db1986 was then further characterized evaluating its nalidixic acid susceptibility by time-kill
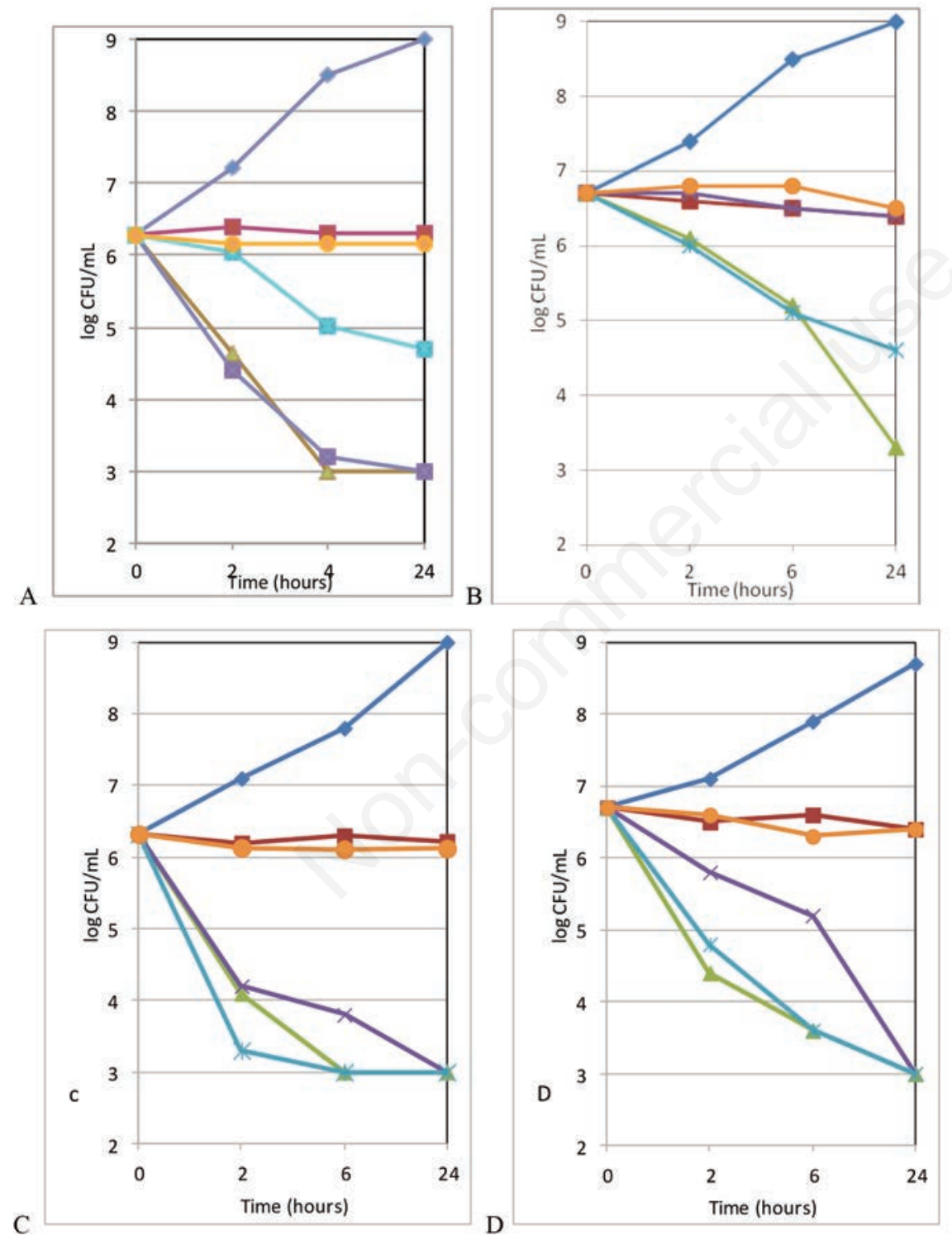

Figure 1. Dynamic bactericidal activity of nalidixic acid and ciprofloxacin with and without chloramphenicol against $\mathrm{C} 600$ and its transductant $\mathrm{db} 1986$ carrying the qsa-1 mutation, under aerobic $(A$ and $C)$ and anaerobic $(B$ and $D)$ conditions. $A$ and $B$ : C600, $C$ and D: db1986. control; $\square$ chloramphenicol $(\mathrm{Cm})(50 \mathrm{mg} / \mathrm{L}) ; \Delta$ ciprofloxacin (Cip) $(0.1$ $\mathrm{mg} / \mathrm{L}$ ); $\mathrm{X}$ nalidixic acid (nal) $50 \mathrm{mg} / \mathrm{L}$. ${ }^{*} \mathrm{Cm}+\mathrm{Cip} ; \bullet \mathrm{Cm}+$ nal. Representative of replicate experiments.

experiment. When, the control strain C600 was exposed to nalidixic acid and ciprofloxacin under aerobic condition a lethal effect was registered, the addition of chloramphenicol, caused a block of the growth in the cultured treated with nalidixic acid but did not abolish the lethal activity of ciprofloxacin (Figure 1A). The same experiments carried out under anaerobic environment demonstrated that ciprofloxacin still had bactericidal activity against this strain while nalidixic acid showed a bacteriostatic effect. The addition of chloramphenicol did not alter the number of survivors showing the same behaviour registered in the bacte-

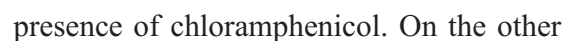
hand nalidixic acid was able to kill $q s a-1$ mutant under these conditions, with the exception in the experimental situations where chloramphenicol was present, in this latter case a bacteriostatic effect was noted (Figure 1D).

\section{Conclusions}

The use of an original method has allowed the isolation of a quinolone (nalidixic acid) susceptible strain under anaerobic environment. The mutant presents some physiological perturbations such as morphological alterations and a long generation time, but in the presence of chloramphenicol the lethal activity of nalidixic acid was still blocked. The chromosomal location of this defect was found in the $\operatorname{trp} A$-topA-cys $B$ region of the $E$. coli genetic map. The mutation was transduced in another host and the transductant behaved like the original mutant, its susceptibility to nalidixic acid was also confirmed by timekill tests.

In considering the present findings it should remind that DNA supercoiling of the bacterial cells is dependent on the aerobic or anaerobic conditions, ${ }^{14}$ in addition other studies reported that strict aerobic or anaerobic mutants are defective in topoisomerase I or gyrase activity respectively. ${ }^{15,16}$

These results lead us to conclude that activity of topoisomerase I, associated with relaxation of chromosomal DNA, is necessary for expression of genes required for aerobic growth, whereas activity of gyrase, associated with supercoiling of chromosomal DNA, is necessary for expression of genes required for anaerobic growth.

Thus the defect in the topoisomerase activity of the mutant found in this study suggests a possible role of DNA supercoiling in the expression of susceptibility to nalidixic acid under anaerobic condition, on the other hand the presence of chloram- 
phenicol block protein synthesis that prevent the production of the ROS pathway responsible of the cell death. ${ }^{11-17}$.

\section{References}

1. Mitscher, L.A. Bacterial topoisomerase inhibitors: quinolone and pyridone Antibact. Agents Chem. Rev. 2005, 105, 559-592.

2. Drlica, K.; Hiasa. H.; Kerns, R.; Malik, M.; Mustaev, A.; Zhao, X. Quinolones: Action and resistance updated. Curr. Top. Med. Chem. 2009, 9:981-998.

3. Aldred, K.J.; Kerns. R,J,; Osheroff, N. Mechanism of quinolone action and resistance. Biochemistry 2014, 53:1565-1574.

4. Hooper, D. C. Mechanisms of action of antimicrobials: Focus on fluoroquinolones. Clin. Infect. Dis. 2001, 32 (5uppl. 1), 59-515.

5. Barbour, S.D. Effect of nalidixic acid on conjugational transfer and expression of episomal lac genes in Escherichia coli K-12. J. Mol. Biol. 1967. 28:373-376.

6. Kreuzer, K.N.; N.R. Cozzarelli. Escherichia coli mutants thermosensitive for deoxyribonucleic acid gyrase subunit A: effects on deoxyribonucleic acid replication, transcription, and bacteriophage growth. J. Bacteriol. 1979, 140, 424-435.

7. Hooper, D.C.; Wolfson, J.; Tung, C.; Souza, K.S.; Swartz, M.N. Effects of Inhibition of the B subunit of DNA gyrase on conjugation in Escherichia coli. J. Bacteriol. 1989, 171: 22352237.

8. Debbia, E.A.; Massaro, S.; Campora. U.; G.C. Schito. Inhibition of F'lac transfer by various antibacterial drugs in Escherichia coli. Microbiologica 1994, 17, 65- 68.

9. Marchese, A.; Debbia, E.A. The role of gyrA, gyrB, and dnaA functions in bacterial conjugation. Ann Microbiol 2016, 66, 223-228.

10. Debbia, E.A,; Marchese, A. Characterization of Hfr strains selected by integrative suppression of F'lac in dnaA, gyrA and gyrB temperature sensitive mutants. J. Biol. Res. 2017; 90:6595 doi:10.4081/jbr. 2017. 6595.

11. Malik, M.; Hussain, S.; Drlica, K. Effect of anaerobic growth on quinolone lethality with Escherichia coli. Antimicrob. Agents Chemother. 2007, 51, 28-34.

12. Miller J.H. Experiments in Molecular Genetics, 1972 Cold 5pring Harbor, New York.

13. Singer, M.; Baker, T.A.; Schnitzler, G.;
Shawn, M.; Deischel, M. G.; Dove, W.; Jaacks, K.J.; Grossman, A.D; Erickson, J.W.; Gross, C. A. A Collection of strains containing genetically linked alternating antibiotic resistance elements for genetic mapping of Escherichia coli Microbiol Rev. 1989. 53: 1-24.

14. Hsieh, Li-S.; Burger, R. M.; Drlica, K. Bacterial DNA supercoiling and [ATP]/[ADP] changes associated with a transition to anaerobic growth J. Mol. Biol. 1991, 219, 443-450.

15. Droffner M.L.; Yamamoto, N. Anaerobic cultures of Salmonella typhimurium do not exhibit inducible proteolytic function of the recA gene and recBC function. J. Bacteriol. 1983.156: 962-965.

16. Yamamoto N.; Droffner, M.L. Mechanisms determining aerobic or anaerobic growth in the facultative anaerobe Salmonella typhimurium. Proc. Natl. Acad. Sci. USA. 1985.82: 2077-2081.

17. Wang, X.; Zhao, X. Malik, M.; Drlica, $\mathrm{K}$. Contribution of reactive oxygen species to pathways of quinolone-mediated bacterial cell death J Antimicrob Chemother 2010; 65: 520-524. 